Session 2793

\title{
A Modular Approach to Vibrations
}

\author{
Sally J. Pardue, Corinne M. Darvennes \\ Tennessee Technological University, Cookeville, Tennessee
}

\begin{abstract}
An undergraduate vibration course has been presented in a modular form to improve student participation and understanding. The new modular format highlights the key concepts and tools required to perform vibration analysis on both single (SDOF) and multiple degree-of-freedom (MDOF) systems. The traditional approach, placing MDOF late in the semester, emphasizes the SDOF model and leaves the students with an oversimplified view of vibrations. A reorganization of the material found in most vibration texts encourages the students to strengthen their system analysis skills.

Module 1 covers the modeling of systems, both SDOF and MDOF. This has been a stumbling block for students thus needing a more focused approach. An early introduction of Lagrange's equations has strengthened students' ability to model complex engineering systems mathematically. Module 2 presents the tools required to carry out future analysis, such as matrix methods, complex notation, and MATLAB. Module 3 encourages physical understanding of the dynamic response of 1 and 2 DOF systems using an air-track demonstration unit. Observing and measuring actual system response motivates the students to understand the upcoming mathematical development. Module 4, the analytical heart of the course, presents free and forced responses for SDOF and MDOF systems. Equations are more easily understood because they correlate to observations made during Module 3. The course ends with Module 5, practical applications.
\end{abstract}

\section{Introduction}

My colleague and I sat on a park bench during Summer 2000 and discussed my recent teaching of our undergraduate vibrations class in Spring 2000. From her experience and mine, we observed overall student understanding of vibrations was disappointing. What were the key issues?

Lack of interest in the subject

Modeling concepts, real systems transformed into SDOF/MDOF models

Application of dynamic principles to obtain equations of motion

Mathematical ability to deal with solution of differential equations

Getting lost in the details 
As we speculated about student interest in the subject, we wondered what could we do to better motivate the students. Perhaps the key to promoting student interest was to present the subject less as static facts and more as a journey or dynamic process in knowledge acquisition. Observe vibration phenomena, recognize need to control or change the phenomena, then search for ways to achieve that control. The student is thus motivated, hopefully, to walk with us through an exploration of the field of vibrations.

\subsection{Real systems to engineering models}

In our past teaching of the subject, the students were introduced to the topic and asked to think about where vibration occurs in their experience. Examples often brought forward are what you feel in vehicle motion, what you hear in music, and machinery applications. Many students had observed a floor vibration in the student study room; below the study room is an equipment room for the building. Structural vibration emanating from air handlers and water pumps feeds uncomfortably into the room above. Students quickly grasped the need to understand what vibration is and further they wanted to know how to be rid of unwanted vibration. For a balanced view, it was also pointed out that vibrations could be desirable phenomena, such as in industrial vibratory polishers. After this intro, we plunged into SDOF systems and followed a standard textbook approach to the subject. There appeared to be confusion in the students mind as to how a SDOF model could be of any use in the "real world."

Problem: Where is the link from a complex system to a SDOF model?

Solution: Spend initial course lectures on system modeling. Demonstrate the process of modeling a real physical system as a mass-spring-damper model.

\subsection{Dynamic principles}

We observed an overall weakness in our student's dynamics background. Given a model as a starting point, the student often had difficulty applying dynamic principles to obtain equations of motion. The free body diagram and Newtonian approach used by most students suffered with sign errors and broke down even more when faced with MDOF systems.

Problem: How to strengthen student understanding and usage of dynamics?

Solution: Immediately following system modeling, present Newtonian and Lagrangian

methods for SDOF and MDOF simultaneously. Implement these concepts on numerous problems.

\subsection{Student Interest and Mathematical ability}

Student interest in the subject tended to drop sharply when faced with the solution of the differential equations. The many vibration scenarios traditionally addressed deal with homogenous and particular solutions to the second-order differential equation subject to various initial conditions and forcing functions. The student often seemed to get lost in detail, not able to recognize the similarity in solution procedures.

Problem: How to overcome student inherent dislike of math and promote continued interest in the subject? 
Solution: Use physical demonstrations of simple one and two degree of freedom systems. Continually point back to these demonstrations when presenting the solution of the differential equations.

\subsection{Getting lost in details}

In general the student view tended to focus on the immediate work, neglecting to put the immediate subject matter into a larger view of the course. We also noted this seems to be a trend not only in this course but in the overall ME program in general. The student wants to compartmentalize the material of each course, not naturally seeking to find how one course relates to another.

Problem: How to promote an attitude focused toward an overall understanding and minimizing the tendency to dwell on specific details?

Solution: Provide a modularized delivery of material with learning objectives for each module clearly stated. Continually demonstrate how these modules are linked thus providing a model for the student to consider when building a global view of all their coursework.

\section{Reorganization of Class Syllabus}

To address the proposed solutions above, we re-examined the past objectives for our course. Previously, our semester course emphasized one degree of freedom, since the course delivery followed the standard organization of most vibrations texts ${ }^{1,2,3,4,5,6}$. It should be noted a new vibration text ${ }^{7}$ provides an organization of the vibration material that coincides with our proposed changes. In our previous teaching, the solution of a SDOF second-order differential equation to free, harmonic, periodic, and transient excitations was addressed in great detail with little student enthusiasm, beginning in the second week of the course. Multi-DOF discussion was limited to 2-DOF in the last weeks of the course and focused primarily on the solution to the eigenvalue problem for natural frequencies and mode shapes. Additionally the course objectives included instruction and usage of MATLAB.

Our new implementation of the course material was as modules. We separated the 15 -week semester into five modules. Initially we allowed three weeks per module, but adjusted the length during planning sessions to accommodate topics. A description of the modules follows.

\subsection{Module 1: Modeling and Dynamics - 3 weeks}

We introduced the course policy and presented the modular layout of the course. Because we were not following the class text in a classic chapter-by-chapter layout, we emphasized to the students our rationale for "jumping around" in the text, hoping to minimize confusion.

Various physical systems and their corresponding dynamic models were presented in the first lectures. We discussed the model elements, lumped mass, springs, and dampers. Coordinate systems, either translational, rotational, or a combination for higher DOF systems were discussed. We talked about the need to have an equation of motion to predict system performance. 
To derive equations of motion, we applied the Newtonian approach of force summation and moment summation to SDOF and 2DOF models. We introduced the Lagrangian method for derivation of equations of motion, specifically to work with higher DOF models. We encouraged students to use both approaches as checks on any given problem.

Important parameters of the SDOF system such as natural frequency and damping ratio were introduced through a generalized form of the equation of motion and identifying the grouping of terms that include these parameters. System parameters were physically interpreted through observations in Module 3.

\subsection{Module 2: Tools -2 weeks}

Our ME students do not take required courses in complex algebra or matrix methods. Since we believe the solution of the differential equations is easier to perform and manipulate in complex notation, we needed to assure that all students in the course had a fundamental exposure to complex algebra. Also, to address MDOF systems, the student had to be familiar with matrix notation and manipulations.

Computer simulations using a tool like MATLAB is also a core component of the class. MATLAB is introduced and although many of the students have some previous experience with the software we want to assure everyone has a core capability. By presenting MATLAB early in the semester, it becomes an integral tool the students can use throughout the course. Subsequent problems often include a MATLAB component.

Demonstration of the matrix techniques was carried out on a 2DOF system. The students were already capable of deriving equations of motion for higher DOF after completing Module 1. Putting the coupled equations into matrix form and then demonstrating the eigensolution followed naturally. The students used MATLAB to solve for natural frequencies and mode shapes using the eig function. They also carried out the analytical solution and were able to see the comparison with MATLAB. In order to set up the analytical solution, the students had to accept some mathematical steps without full understanding, such as the assumed solution form of harmonic motion for free response.

\subsection{Module 3: Physical Demonstrations - 3 weeks}

The planned inclusion of a demonstration unit in the classroom for multiple class periods provided for formal observation time. The students could see and measure a SDOF and 2DOF system in motion. The approach of this module was to allow the students to observe vibration phenomena and then to describe it in their own terms prior to the full mathematical treatment of Module 4.

The demonstration unit is an air-track system shown in Figure 1. We placed accelerometers on the masses and recorded the response signals for various excitations; free vibration with initial displacement, initial velocity via an impact, and base excitation through a motor and scotch-yoke 


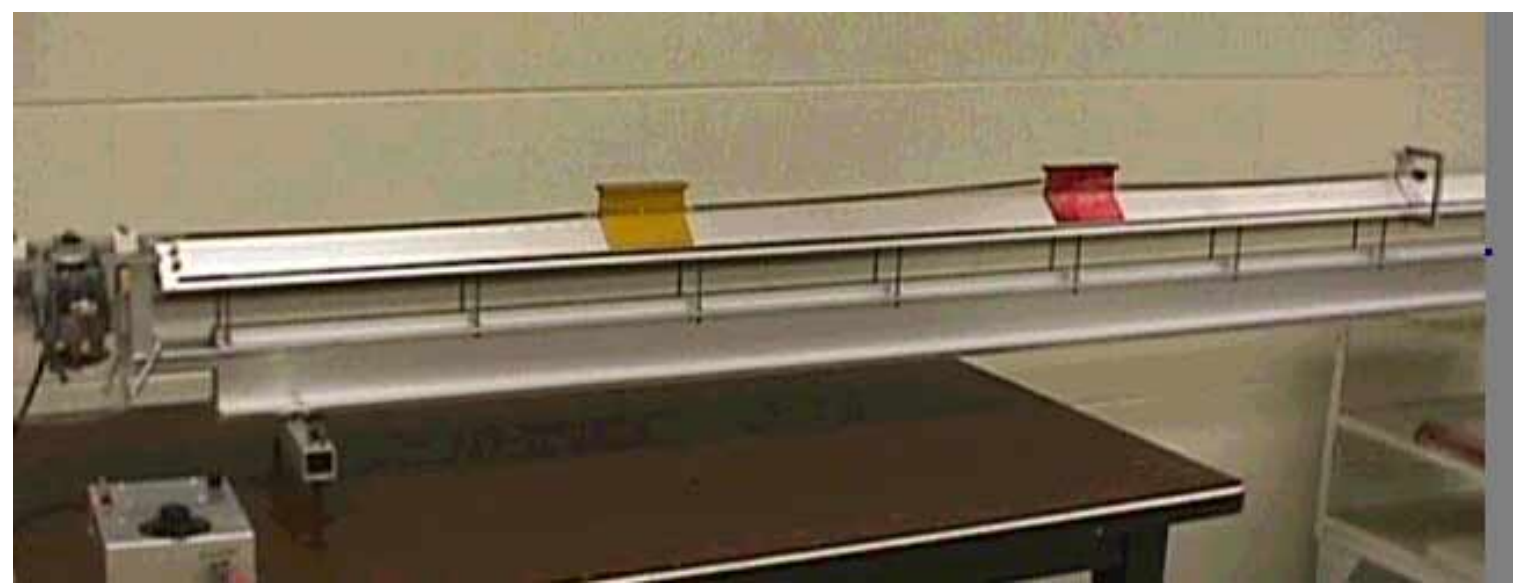

Figure 1. Air track demonstration unit, 2DOF system set-up with motor input at left

mechanism. The signal was displayed on a Tektronix digital scope and projected onto the screen via an overhead projector and camera system.

Example demo: On a SDOF setup, we measured free response to a displacement input. We asked the students to describe what they saw on the oscilloscope and they named the sine wave. Thus the harmonic response that is always assumed mathematically became real for them. We measured period and demonstrated how to change this into natural frequency of the system. Informing the students of the mass, we asked them to determine spring value. The known spring value was then compared to the value obtained via the performed measurement. Invariably a mismatch occurs, and the students were prompted to speculate as to why.

With two mass values and various springs for the system, many configurations are possible. In subsequent demos, we challenged students to predict outcomes due to changes in the system. Whether they predicted correctly or incorrectly we were able to discuss in class how they reached these conclusions. Student enthusiasm for and participation in class discussion was notably active.

The demonstration phase was also an excellent place to introduce the concept of time domain and frequency domain. The demonstration system is driven with harmonic base input by a motor and scotch-yoke mechanism. On a SDOF setup, we change the motor speed and gradually drive the system into resonance. The students are asked to describe what is taking place. We could map out the displacement of the mass for various motor inputs and trace out the response curve as a function of driving frequency, laying the groundwork for the classic presentation of system response plotted against frequency ratio as given in standard vibration texts.

\subsection{Module 4: Differential Equation Solutions - 5 weeks}

Analytical time domain solutions of the SDOF equation of motion were presented for free vibration, harmonic excitation, and transient excitation. The concepts of superposition solution and Lap lace transform were also used. Although the students have a prerequisite math course in differential equations, they are decidedly unsure of solution procedures. 
A 2DOF system was solved for the time domain response. Since the eigensolution had already been addressed in Module 2, the students focused on the process for solving coupled differential equations via a direct approach. The modal solution technique is reserved for a follow-on option course in vibrations.

Numerical solutions to differential equations were presented using MATLAB and the ode23 function. For MDOF systems, the student was introduced to the state space representation. A comparison of numerical solution to analytical solution was made.

\subsection{Module 5: Applications - 2 weeks}

The two applications we focused on were vibration isolation, a SDOF design problem, and vibration absorbers, a 2DOF design problem. By placing these applications at the close of the course, we were able to review all the previous modules and their impact on these design problems. We also discussed the overall results of each approach and where each technique for reducing vibration might be appropriate.

\subsection{Observations and Recommendations}

The modularized version of vibrations was presented to two sections of ME305 Vibrations in Fall 2000. Dr. Darvennes taught one section and Dr. Pardue the other. Quantitative measures of improved student performance due to the modular delivery of the material were not undertaken. However, we do offer qualitative comments.

\subsection{Modular organization of course}

In the future, Module 2(Tools) and Module 3(Physical Demonstrations) will be reversed. The Physical Demonstrations should directly follow Module 1(Modeling and Dynamics). The student will observe early in the semester the frequently used harmonic assumption for response employed in both the eigenvalue problem and the time solution of the differential equation of motion. Also, the 2DOF system in the Tools Module will be better supported since the students will have already observed a system response involving two frequencies occurring simultaneously. Also, mode shapes will have been presented in the demonstration module.

The learning objectives for each module were presented at the beginning of the semester and at the start of each module. Yet student criticism at the close of the course included comments regarding the clarity of course delivery. They were unsure of what they learned and in what order; indeed they had difficulty in global zing the facts, obviously staying detail oriented.

To address these student concerns, the learning objectives will be presented on a weekly (or perhaps daily) lecture basis, showing how the immediate information fits into the larger scope. A visual cue may be employed to enhance the student perception.

The length of the Physical Demonstrations Module will be reduced from 3 weeks to 2 weeks. We found three weeks was too long and six class periods should be sufficient to demonstrate the SDOF and 2DOF systems. We plan to bring out the demonstration air-track again as needed

"Proceedings of the 2001 American Society for Engineering Education Annual Conference \& Exposition Copyright C 2001, American Society for Engineering Education” 
during the Module 4 (Differential Equations Solutions) to remind students visually of what they observed early in the semester. The student interest level should also receive a boost by observing the demonstrations in shortened form again. The week removed from the Demonstrations Module will be added to Module 5 (Applications). A third week on Applications will allow for more in-class group work sessions during the final week of the class. This will serve as a course review and promote student participation in the review process.

\subsection{Improved Modeling and Dynamic Capability}

After the initial lectures regarding modeling, the students were not asked to develop their own models from actual physical systems. The starting point for most problems they were assigned was a verbal description of a model or a model schematic. A larger repertoire of real systems modeled as SDOF or MODF is needed. The students could provide examples from their experience with the class determining models through in-class group work.

The students were required throughout the semester to identify from given schematics what coordinates and how many DOF were appropriate. The students were assigned numerous problems deriving equations of motion either using Newtonian, Lagrangian, or their choice. Some class periods were dedicated to in-class problem solving among student groups. This was very beneficial to the instructor roaming through the room acting as a resource to the groups. Often misconceptions came to light and could be corrected "real-time" rather than later through homework or exams.

A general comparison of exam results from previous semesters indicated an improved capability regarding the derivation of equations of motion using Newtonian and Lagrangian concepts.

\subsection{Mathematical ability and student interest}

It is difficult to ascertain whether the reorganization of material significantly improved the mathematical ability of the students. Overall, they are not enthusiastic in their participation during this phase of the course material. However, it was beneficial to remind them of the physical demonstrations we had seen when they began to get lost in the process of analytical solution.

\subsection{Conclusions}

Overall, we found the modular approach to presenting a semester or 15 weeks of vibration material an effective delivery tool. We found student interest was improved especially during the modeling discussions and during the physical demonstrations. Student performance on deriving equations of motion was better than previous semesters where SDOF had been emphasized with MDOF occurring late in the semester. By considering MDOF systems from the beginning of the course the students had greater exposure to the process of sorting problem type and they felt better connected to "real-world" systems. 


\section{Bibliography}

1. Inman, D.J., Engineering Vibration, $2^{\text {nd }}$ ed., Prentice Hall, New Jersey, 2001.

2. Tongue, B. H., Principles of Vibration, Oxford, University Press, New York, 1996.

3. Kelly, S. G. Fundamentals of Mechanical Vibrations, McGraw-Hill, New York, 2000.

4. James, M. L., et al, Vibration of Mechanical and Structural Systems, HarperCollins, New York, 1994.

5. Thomson, W. T., and Dahleh, M.D., Theory of Vibration and Applications, Prentice Hall, New Jersey, 1998.

6. Rao, S.S., Mechanical Vibrations, $3^{\text {rd }}$ ed., Addison-Wesley, Massachusetts, 1995.

7. Ginsberg, J. H., Mechanical and Structural Vibrations, Wiley, New York, 2001.

\section{SALLY PARDUE}

Sally Pardue is an Assistant Professor of Mechanical Engineering at Tennessee Technological University. She began her academic appointment in August 1999 following four years as a Research and Development Engineer. Dr. Pardue received her PhD in Engineering from Tennessee Technological University in 1995. Her research interests are random vibrations and machine diagnostics.

\section{CORINNE DARVENNES}

Corinne Darvennes is an Associate Professor of Mechanical Engineering at Tennessee Technological University. Dr. Darvennes received a Diplôme d'Ingénieur of Mechanical Engineering from Universit de Technologie de Compi gne, France, in 1984, an MSc from the Institute of Sound and Vibration Studies, Southampton, England, in 1985, and a PhD in Mechanical Engineering from The University of Texas at Austin in 1989. In 2000, she became an INCE Board Certified Noise Control Engineer. 